\title{
Lipoprotein glomerulopathy induced by ApoE Kyoto mutation in ApoE-deficient mice
}

\author{
Hongyan Wu' ${ }^{1}$, Jing Yang ${ }^{1}$, Yun-Qiang Liu², Song Lei ${ }^{3}$, Mei Yang ${ }^{3}$, Zhi Yang ${ }^{1}$, Yuan Yang ${ }^{2}$ and Zhangxue Hu ${ }^{1 *}$ (i)
}

\begin{abstract}
Background: Lipoprotein glomerulopathy $(L P G)$ is a rare autosomal dominant kidney disease that is most commonly caused by mutations in ApoE Kyoto (p.R43C) and ApoE Sendai (p.R163P). Differences in phenotype among the various ApoE mutations have been suggested, but the pathogenic role of ApoE Kyoto has not been validated in an animal model. This study intended to establish an ApoE Kyoto murine model and to further compare the pathologic differences between ApoE Kyoto and ApoE Sendai.

Method: Male ApoE-deficient mice, 3 months of age, were divided into five groups, including the AD-ApoE Sendai, AD-ApoE Kyoto, AD-ApoE3, AD-eGFP, and ApoE (-/-) groups. The first four groups received recombinant adenovirus that contained the entire coding regions of the human ApoE Sendai and ApoE Kyoto, apoE3, and EGFP genes, respectively. Fasting blood and urine samples were collected at multiple time points. Lipid profiles and urine albumin-creatinine ratio were measured. Renal and aortic histopathologic alterations were analyzed.

Results: After virus injection, plasma human ApoE was detected and rapidly reached the maximum level at 4-6 days in the AD-ApoE Kyoto and AD-ApoE Sendai groups $(17.4 \pm 3.1 \mu \mathrm{g} / \mathrm{mL}$ vs.: $22.2 \pm 4.5 \mu \mathrm{g} / \mathrm{mL}$, respectively) and at 2 days in the AD-ApoE3 group $(38.4 \mu \mathrm{g} / \mathrm{mL})$. The serum total cholesterol decreased by $63 \%, 65 \%$, and $73 \%$ in the AD-ApoE Kyoto, AD-ApoE Sendai and AD-ApoE3 groups, respectively. There were no significant changes in serum triglyceride and urinary albumin-creatinine ratio among the five groups. Typical lipoprotein thrombi with positive ApoE staining were detected in the AD-ApoE Kyoto and AD-ApoE Sendai groups. The Oil-red O-positive glomerular area tended to be higher in the AD-ApoE Kyoto group (9.2\%) than in the AD-ApoE Sendai (3.9\%), AD-ApoE3 (4.8\%), AD-eGFP (2.9\%), and $\mathrm{ApoE}(-/-)$ (3.6\%) groups. The atherosclerotic plaque area in the aorta was lower in the group injected with various ApoE mutations than in the group without injection of ApoE mutation.
\end{abstract}

Conclusions: In this animal study, we first established an ApoE Kyoto mutation murine model and confirmed its pathogenic role in LPG. Our results suggested that LPG may be more severe with the ApoE Kyoto than with the ApoE Sendai.

Keywords: Lipoprotein glomerulopathy, ApoE Kyoto (p.R43C), ApoE Sendai (p.R163P), Atherosclerosis, Recombinant adenovirus

\footnotetext{
*Correspondence: hzxawy@scu.edu.cn

1 Department of Nephrology and National Clinical Research Center for Geriatrics, West China Hospital, Sichuan University, Sichuan, Guoxue Alley, 37\#, Wuhou District, Chengdu 610041, China

Full list of author information is available at the end of the article
}

\section{Background}

Lipoprotein glomerulopathy (LPG) is a rare autosomal dominant kidney disease with incomplete penetrance. To date, more than 200 patients with LPG have been reported worldwide and were mainly of Japanese and Chinese origin [1]. The presence of massive lipoprotein thrombi containing ApoE in dilated glomerular papillary lumina is the typical pathologic manifestation $[2$,

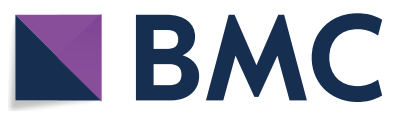

(c) The Author(s) 2021. This article is licensed under a Creative Commons Attribution 4.0 International License, which permits use, sharing, adaptation, distribution and reproduction in any medium or format, as long as you give appropriate credit to the original author(s) and the source, provide a link to the Creative Commons licence, and indicate if changes were made. The images or other third party material in this article are included in the article's Creative Commons licence, unless indicated otherwise in a credit line to the material. If material is not included in the article's Creative Commons licence and your intended use is not permitted by statutory regulation or exceeds the permitted use, you will need to obtain permission directly from the copyright holder. To view a copy of this licence, visit http://creativeco mmons.org/licenses/by/4.0/. The Creative Commons Public Domain Dedication waiver (http://creativecommons.org/publicdomain/ zero/1.0/) applies to the data made available in this article, unless otherwise stated in a credit line to the data. 
3]. Nephrotic-range proteinuria, hypertriglyceridemia, and progressive renal dysfunction accompanied by elevated ApoE level are common among most patients, and half of the patients may eventually develop uremia in 1-27 years. It is interesting that cardiovascular events such as angina pectoris, myocardial infarction et al. were rarely reported in LPG patients despite abnormal lipid profiles. Renal pathological findings are crucial to diagnosis, since LPG patients with normal lipid profiles have been reported [4-6].

Pathogenesis of LPG involves ApoE mutation and dysfunction of macrophage although detailed mechanism remains unknown. Human ApoE is a $34-\mathrm{kDa}$ protein with 299 amino acids and is encoded by the $A p o E$ gene $(A p o E)$, which is located at $19 \mathrm{q} 13.2$ and has four exons and three introns [7]. It mediates the tissue uptake of triglyceride-rich lipoproteins through both the low-density lipoprotein receptor (LDLR) and the LDL receptorrelated protein pathways. Moreover, ApoE is a ligand for heparan sulfate proteoglycan. To date, 18 ApoE variants $[1,8]$ have been reported to be associated with LPG. ApoE Sendai (p. Arg163Pro), which is located in the LDLR binding area, was the first reported ApoE mutation related with LPG in East Japan [9]. Yamamoto et al. proved the pathogenicity of ApoE Sendai by developing LPG in ApoE-deficient mice using virus-mediated transduction of ApoE Sendai [10]. ApoE Kyoto (p. Arg43Cys), which is another leading ApoE variant that is related to LPG, is located outside LDLR binding area and has been reported worldwide. Previously, we reported the clustering of LPG patients carrying the ApoE Kyoto in Sichuan province, China [2]; this has been the largest reported group of LPG until now. Matsunaga et al. showed that the binding ability of recombinant apoE3 Kyoto to LDLR was reduced to $10 \%$, compared with that of recombinant apoE3, in vitro [11]. However, the role of ApoE Kyoto has not been validated in animal model. A recent study showed that patients with ApoE mutations in the LDLR binding area may have relatively high blood pressure and serum ApoE [12], suggesting phenotype differences among various ApoE mutations. In this study, we developed an LPG model in ApoE knockout mice by injecting adenovirus carrying ApoE variants, including $A P O E$ Kyoto, APOE Sendai, and APOE3, and further compared the laboratory and histopathologic differences among the LPG mice with various ApoE mutations.

\section{Methods}

\section{Recombinant adenoviruses construction}

Recombinant adenoviruses AD-apoE3, AD-ApoE Sendai, AD-ApoE Kyoto, and AD-eGFP containing the entire coding regions of human apoE3, ApoE Sendai, ApoE Kyoto, and enhanced green fluorescent protein were packaged by GeneChem Co., Ltd., (Shanghai, China). All transgene expressions were under the control of the immediate early promoter of cytomegalovirus. Viruses were purified by double cesium chloride gradient ultracentrifugation, and viral titer was determined by plaque assay and expressed as plaque-forming units (pfu). Purified virus aliquots were stored at $-80^{\circ} \mathrm{C}$.

\section{Vector validation in cultured HepG2 and 293 T cells}

In order to estimate the adenoviral gene transfection and expression efficiency in vitro, HepG2 and $293 \mathrm{~T}$ cells were infected with four recombinant adenoviruses at multiplicity of infection (MOI) of 40 and 4, respectively. After incubation for $48 \mathrm{~h}$, the GFP expression and infection efficiency were determined under fluorescence microscopy. The cell protein and growth medium were collected for Western blot and ELISA analyses. The protein of the transfected HepG2 and 293 T cells were extracted by a protein extraction kit (Bio Teke Corporation, China), and the protein concentration was determined by the BCA Protein Assay Kit (Bio Teke Corporation, China), according to the manufacturer's protocol. Thereafter, total protein $(15 \mu \mathrm{g})$ was loaded into each lane of acrylamide gel and subjected to SDS-PAGE. The membranes were incubated with antibodies against human ApoE (ab183597, Abcam) at $4{ }^{\circ} \mathrm{C}$ overnight; $\alpha$-tubulin antibody (sc-8035, Santa Cruz Biotechnology) was used as an internal control to verify the basal expression level and equal protein loading. Target protein bands were captured by the chemiluminescence imager (iBright CL1000, Thermo Scientific).

\section{Reverse transcription quantitative polymerase chain reaction (RT-qPCR)}

Total RNA was extracted using RNA extraction kit (Bio Teke Corporation, China), according to the manufacturer's instructions. The reverse transcription of total RNA into cDNA was performed according to the instructions of HiScript ${ }^{\circledR}$ II Q RT SuperMix (R223-01, Vazyme, China). The TB Green ${ }^{\mathrm{TM}}$ Premix Ex Taq ${ }^{\mathrm{TM}}$ II kit (TaKaRa Bio, Dalian, China) was used for the qPCR. The results were performed using the CFX96 ${ }^{\mathrm{TM}}$ real-time PCR detection system (Bio-Rad, USA), which had the following program design: denaturation step at $95^{\circ} \mathrm{C}$ for $30 \mathrm{~s}$, followed by 40 cycles of denaturation at $95{ }^{\circ} \mathrm{C}$ for $5 \mathrm{~s}$, annealing at $62{ }^{\circ} \mathrm{C}$ for $30 \mathrm{secs}$, and extension at $72{ }^{\circ} \mathrm{C}$ for $30 \mathrm{~s}$. The sequences of the primer pairs were as follows: GAPDH-F: ACGGATTTGGTCGTATTGGG; GAPDH-R: CGCTCC TGGAAGATGGTGAT; $A p o E-F$ : CACAGGCAGGAA GATGAAGGTT; and ApoE-R: TAATCCCAAAAGCGA CCCAGT. The relative amount of genes for internal control was calculated using the comparative $2^{-\Delta \Delta C T}$ method and was normalized to that of GAPDH. 


\section{Animal model establishment}

Male ApoE (-/-) mice on a C57BL/6 background were purchased from Beijing Vital River Laboratory Animal Technology Co., Ltd., and genotype was determined before experiment. All animals were bred and housed at the animal experiment center of Sichuan University and were maintained on normal chow diet ( $4 \%$ fat, $0 \%$ cholesterol). Twenty-seven weight- and age-matched (3 months of age) male ApoE (-/-) mice were randomly divided into five groups, as follows: AD-ApoE Sendai $(n=6)$, AD-ApoE Kyoto $(n=6)$, AD-ApoE3 $(\mathrm{n}=6)$, AD-eGFP $(\mathrm{n}=5)$, and ApoE $(-/-)(\mathrm{n}=4)$. At multiple time points (baseline, and 2, 4, 6, 10, 14, 20, $34,50,65,90$ days after adenoviruses injection), blood was collected from the retroorbital venous plexus after a four-hour fasting. Plasma total cholesterol and triglyceride were detected by enzymatic assay kits (Wako Pure Chemical Co., Osaka, Japan). Accurate quantification of human ApoE in mouse plasma was determined by sandwich ELISA kit (Cat \# EHAPOE, Thermo scientific). Urinary albumin was measured using a mouse albumin ELISA Quantification kit (Bethyl Laboratories, Montgomery, TX). Urine creatinine was determined by a creatinine assay kit (DICT-500, BioAssay System), according to the protocols of the manufacturer. The urine albumin-creatinine ratio (ACR) was calculated to estimate the amount of proteinuria.

\section{Histologic analysis}

Kidneys were dissected after 90 days of virus injection. Renal sections were fixed in $4 \%$ paraformaldehyde, embedded in paraffin, and deparaffinized in xylene. Thereafter, the sections were stained with hematoxylin/ eosin and periodic acid-Schiff. The rabbit antihuman ApoE polyclonal antibody (ab24139, Abcam) was used for immunofluorescence staining, which followed the standard protocol. Frozen sections were stained with Oil-red O. All the Oil-red O-positive glomeruli of five groups were captured, then the area was calculated by Image J software, the positive area ratio of each glomerulus and the percentage of positive glomeruli in five groups were analyzed finally. A small block of the kidney specimen was fixed with $2.5 \%$ glutaraldehyde for electron microscopy detection. Serial 5 - $\mu \mathrm{m}$-thick cryosections of the aortic valve were mounted on masked slides and stained with Oil-red O, the Oil-red O positive atherosclerotic plaque area and the area difference of aorta adventitia and intima in the aortic valve were quantified by the Motic images Advanced 3.2 software and the ratio of two parameters was described as atherosclerotic plaque area percentage.

\section{Statistical analysis}

GraphPad prism 6 software was used to carry out statisti$\mathrm{cal}$ analyses, and all data were presented as mean $\pm \mathrm{SD}$. One-way analysis of variance test was used to compare the mean values between five groups, statistical significance for all comparisons was assigned at $p<0.05$.

\section{Results \\ Transfection efficiency and in vitro validation of recombinant adenovirus}

After transfecting the HepG2 and $293 \mathrm{~T}$ cells with four different recombinant adenoviruses, approximately $80 \%$ to $85 \%$ of the cells expressed eGFP at $48 \mathrm{~h}$ after infection. Western blot and qPCR analysis demonstrated that human ApoE was overexpressed in the cells infected with AD-apoE3, AD-ApoE Sendai, and AD-ApoE Kyoto but not in the cells infected with AD-eGFP (Fig. 1). In addition, the ELISA analysis of the cellular supernatant indicated that, at the same MOI, more human ApoE protein was secreted in the Ad-apoE3 group than in the other groups. On the other hand, no human ApoE was detected in the AD-eGFP group (Additional file 1: Table S1).

\section{Quantification of human ApoE in the plasma of ApoE (-/-) mice}

In the $\mathrm{AD}-\mathrm{ApoE} 3$ group, the expression of plasma human ApoE rapidly reached a peak of $38.4 \mu \mathrm{g} / \mathrm{mL}$ on the $2 \mathrm{nd}$ day after virus injection and declined to a low level on the 10th day. In the AD-ApoE Sendai and AD-ApoE Kyoto groups, human ApoE was detected on the 2nd day; reached the peak levels of $22.2 \mu \mathrm{g} / \mathrm{mL}$ and $17.4 \mu \mathrm{g} / \mathrm{mL}$, respectively, after 4-6 days; and declined to the baseline level on the 10th day. There were no significant differences in the ApoE peak concentration between the ADApoE Kyoto and AD-ApoE Sendai groups. In addition, no ApoE was detected in the AD-eGFP and ApoE (-/-) groups (Fig. 2).

\section{Plasma total cholesterol and triglyceride and urine albumin-creatinine ratio detection}

Among the normal chow-fed ApoE (-/-) mice, the mean baseline total cholesterol levels before virus injection were $416.7 \pm 81.7 \mathrm{mg} / \mathrm{dL}$ in the AD-ApoE Kyoto group, $406.9 \pm 29.1 \mathrm{mg} / \mathrm{dL}$ in the AD-ApoE Sendai group, $521 \pm 50.6 \mathrm{mg} / \mathrm{dL}$ in the AD-ApoE3 group, $403.6 \pm 50.9 \mathrm{mg} / \mathrm{dL}$ in the AD-eGFP group, and $316.8 \pm 26.4 \mathrm{mg} / \mathrm{dL}$ in the ApoE (-/-) group. Along with the expression of human ApoE, plasma total cholesterol began to decrease to the lowest level at 4-6 days after virus injection and gradually increased to baseline thereafter. The relative reduction in total cholesterol level was $63 \%, 65 \%$, and $73 \%$ in the AD-ApoE Kyoto, AD-ApoE 
$\mathbf{a}$
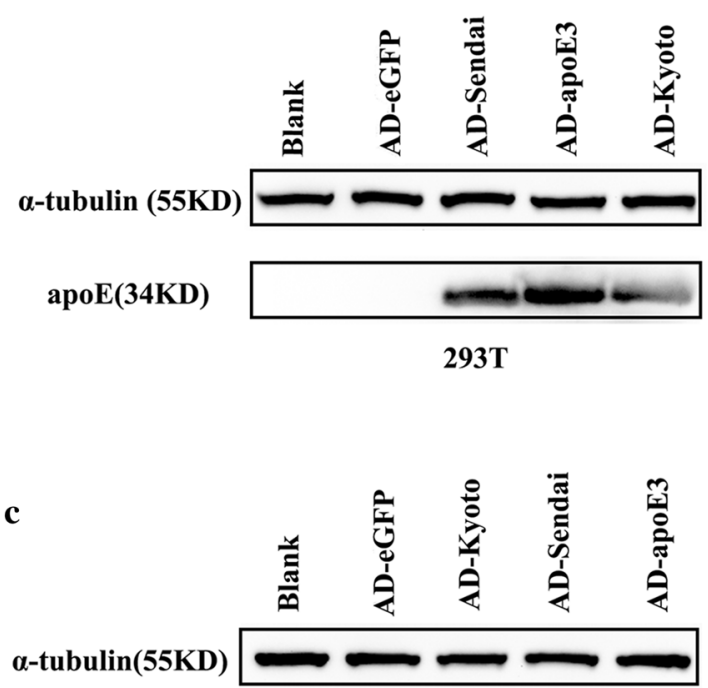

$\operatorname{apoE}(34 K D)$

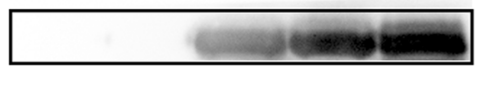

HepG2 b

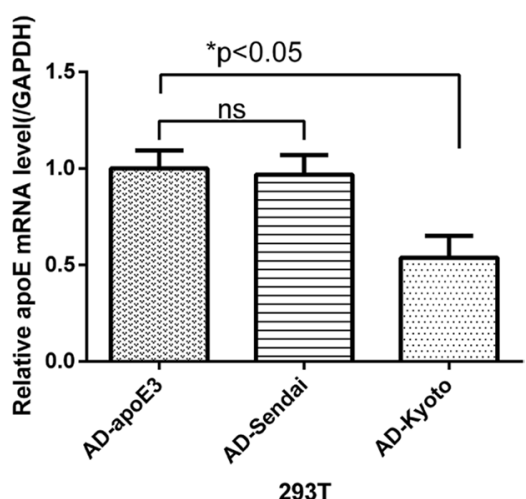

d

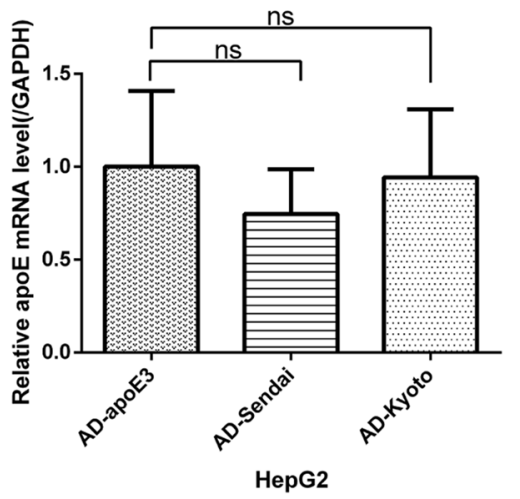

Fig. 1 Overexpression of various human ApoE in 293 T and HepG2 cells induced by recombinant adenovirus transfection. Western blot and qPCR analysis show that human ApoE is overexpressed in the AD-apoE3, AD-ApoE Kyoto, and AD-ApoE Sendai groups but is absent in the AD-eGFP and blank groups

Sendai, and AD-ApoE3 groups, respectively. There were no apparent changes in total cholesterol in the AD-eGFP and ApoE (-/-) groups.

Among the normal chow-fed ApoE (-/-) mice, the mean baseline triglyceride levels before virus injection were $187.7 \pm 48.4 \mathrm{mg} / \mathrm{dL}$ in the AD-ApoE Kyoto group, $197 \pm 41.8 \mathrm{mg} / \mathrm{dL}$ in the AD-ApoE Sendai group, $178.6 \pm 31.4 \mathrm{mg} / \mathrm{dL}$ in the $\mathrm{AD}$-apoE3 group, $170.7 \pm 52.4 \mathrm{mg} / \mathrm{dL}$ in the AD-eGFP group, and $188.4 \pm 37.5 \mathrm{mg} / \mathrm{dL}$ in the ApoE (-/-) group. Unlike the total cholesterol, the triglyceride and urine ACR levels had no apparent changes in the five groups during the observation period (Fig. 2).

The mean plasma total cholesterol and triglyceride in male wild mice on a C57BL/6 background from the same company were $95.39 \pm 22.78 \mathrm{mg} / \mathrm{dl}$, and $126.4 \pm 25.2 \mathrm{mg} /$ $\mathrm{dl}$, respectively.

\section{Histologic manifestation of renal lesions}

The renal lesions in the five groups were estimated 90 days after adenovirus injection, as shown in Fig. 4. LPG-like renal lesions were observed in three mice of the AD-ApoE Kyoto group and in one mouse of the ADApoE Sendai group. Lipoprotein thrombi were observed in the dilated capillary lumina and mesangial area of the glomeruli by toluidine blue staining and were positive for ApoE and Oil-red $\mathrm{O}$ staining. Under electron microscopy, the thrombus-like substances comprised osmiophilic granules and lipid vacuoles of various sizes (Fig. 3). The typical renal lesions in the AD-ApoE Kyoto group are demonstrated in Fig. 4. There were no typical lipoprotein thrombi observed in the other three groups. The Oil-red O-positive glomerular area in $\mathrm{AD}$-ApoE Kyoto group was $9.2 \%$ and tended to be higher, compared with that in the other groups [3.9\% in AD-ApoE Sendai, $4.8 \%$ in AD-apoE3, 2.9\% in AD-eGFP, and 3.6\%, in ApoE (-/-) groups]. There was no difference in the Oil-red O-positive glomeruli percentage among the groups (Fig. 5).

\section{Analysis of atherosclerosis in the aortic valve}

Among the ApoE-deficient mice with normal chow diet, the mean atherosclerotic plaque area in the aortic root was $0.024 \pm 0.008 \times 10^{6}{\mu m^{2}}^{2}$. Three months later, the atherosclerotic plaque increased in size and 

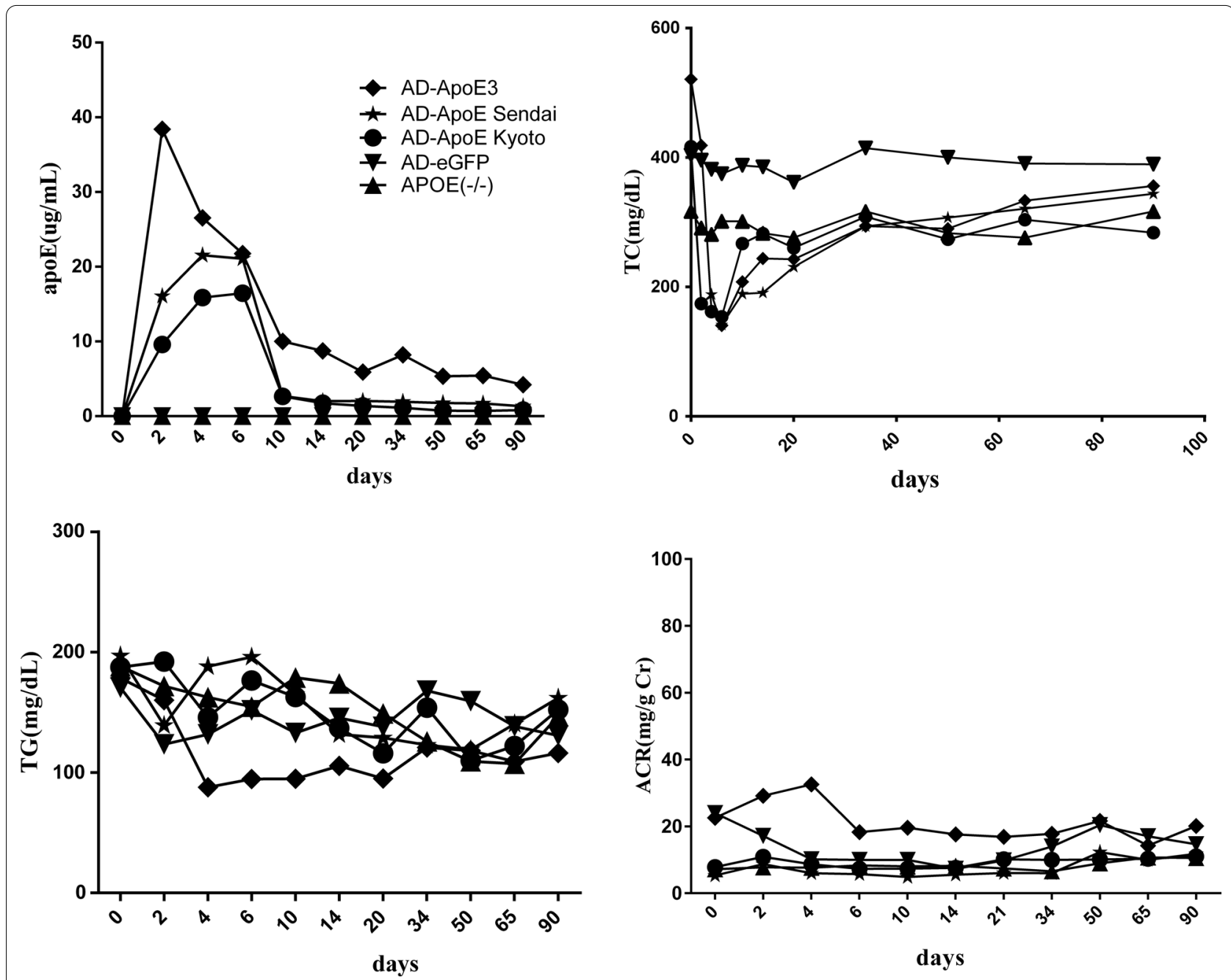

Fig. 2 Alterations in the serum ApoE, total cholesterol (TC), total triglyceride (TG), and urinary albumin-creatinine ratio (ACR) in the five groups

measured $\quad 0.18 \pm 0.05 \times 10^{6} \quad \mu \mathrm{m}^{2}, \quad 0.16 \pm 0.06 \times 10^{6}$ $\mu \mathrm{m}^{2}, \quad 0.13 \pm 0.06 \times 10^{6} \mu \mathrm{m}^{2}, \quad 0.25 \pm 0.1 \times 10^{6} \mu \mathrm{m}^{2}$, and $0.27 \pm 0.15 \times 10^{6} \mu \mathrm{m}^{2}$ in the AD-ApoE Kyoto, AD-ApoE Sendai, AD-apoE3, AD-eGFP, and ApoE (-/-) groups, respectively (Fig. 6). Atherosclerosis progression was slower in the three groups that were injected with various ApoE variants than in the other two groups.

Oil red $\mathrm{O}$ staining results in lung from AD-ApoE Sendai, AD-ApoE Kyoto and $\mathrm{ApoE}(-/-)$ groups. There are thrombi-like material in pulmonary capillaries. However, there are no differences among them (Additional file 2: Figure S1).

\section{Discussion}

LPG is a rare inherited renal disease characterized by formation of intraglomerular lipoprotein thrombi secondary to lipid deposition in the lumina of severely dilated glomerular capillaries. The typical clinical manifestations of LPG include proteinuria or nephrotic syndrome and elevated serum ApoE. ApoE Kyoto has been the most common mutation in LPG. In this study, we first established an LPG murine model induced by ApoE Kyoto. Thrombi in dilated glomerular capillaries with positive staining for ApoE and Oil-red $O$ were observed in the AD-ApoE Kyoto and AD-ApoE Sendai groups and were seen to comprise osmiophilic granules and lipid vacuoles of various sizes on electron microscopy. All of the above supported the pathogenicity of ApoE Kyoto in LPG.

Notably, the lipoprotein thrombi area seemed larger in the ApoE Kyoto mice than in the ApoE Sendai mice, although the lipid profiles of both groups were comparable. ApoE Kyoto and ApoE Sendai both have significantly reduced helical content and are thermodynamically destabilized and prone to aggregation [13]. ApoE Sendai is located in the LDLR binding area, and the affinity 


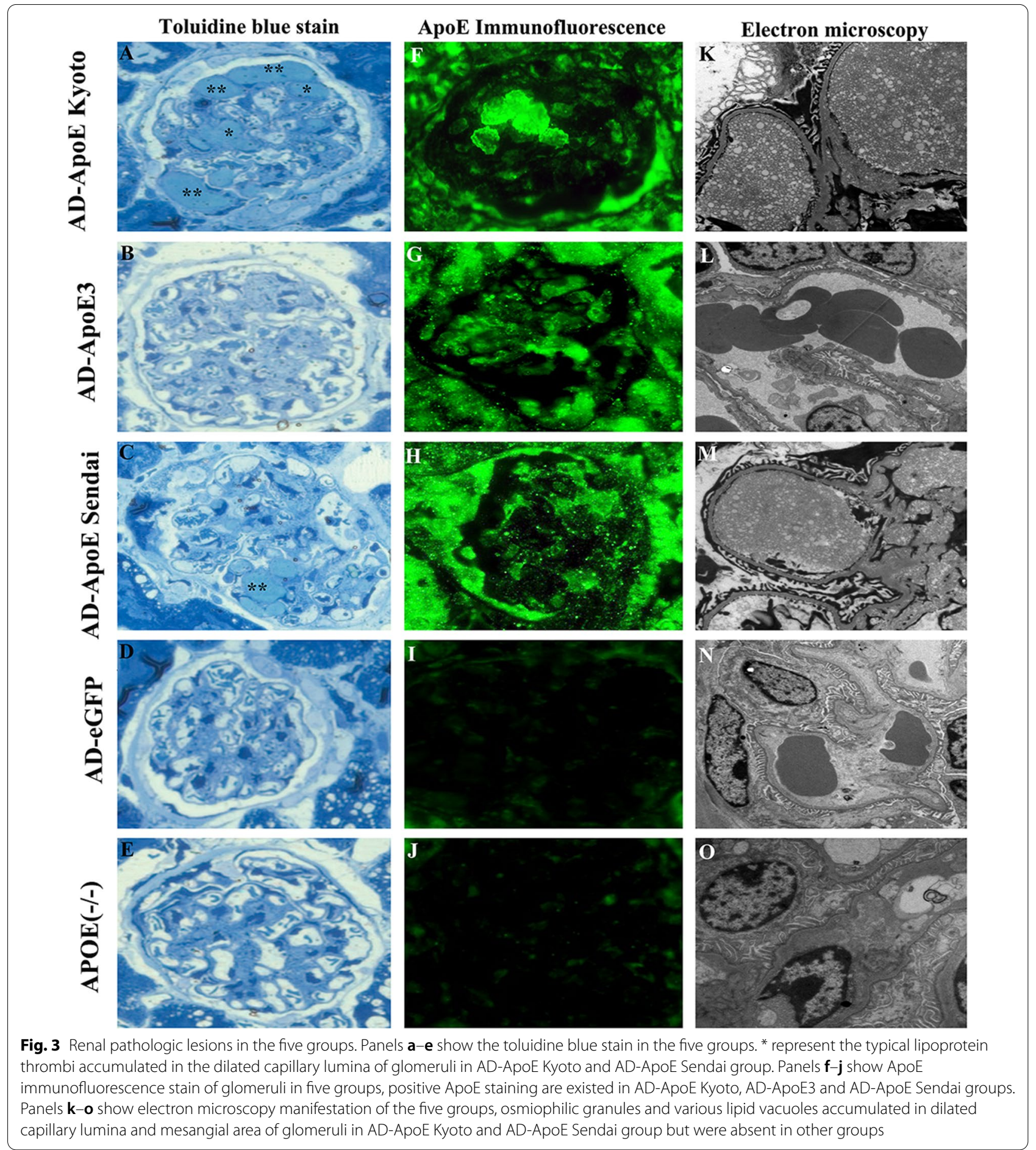

of ApoE Sendai for LDLR was decreased [10]. Recently, patients with LPG with mutations in the LDL receptor binding region were reported to have relatively high blood pressure and serum ApoE levels [12]. However, detailed pathologic analyses were lacking. Although
ApoE Kyoto has reduced affinity for LDLR, it is located outside the LDLR binding area. Furthermore, triglyceride-rich lipoprotein containing ApoE Kyoto had been shown to have increased affinity for endothelial cells, and this may be manifested as local adhesion [14]. In 

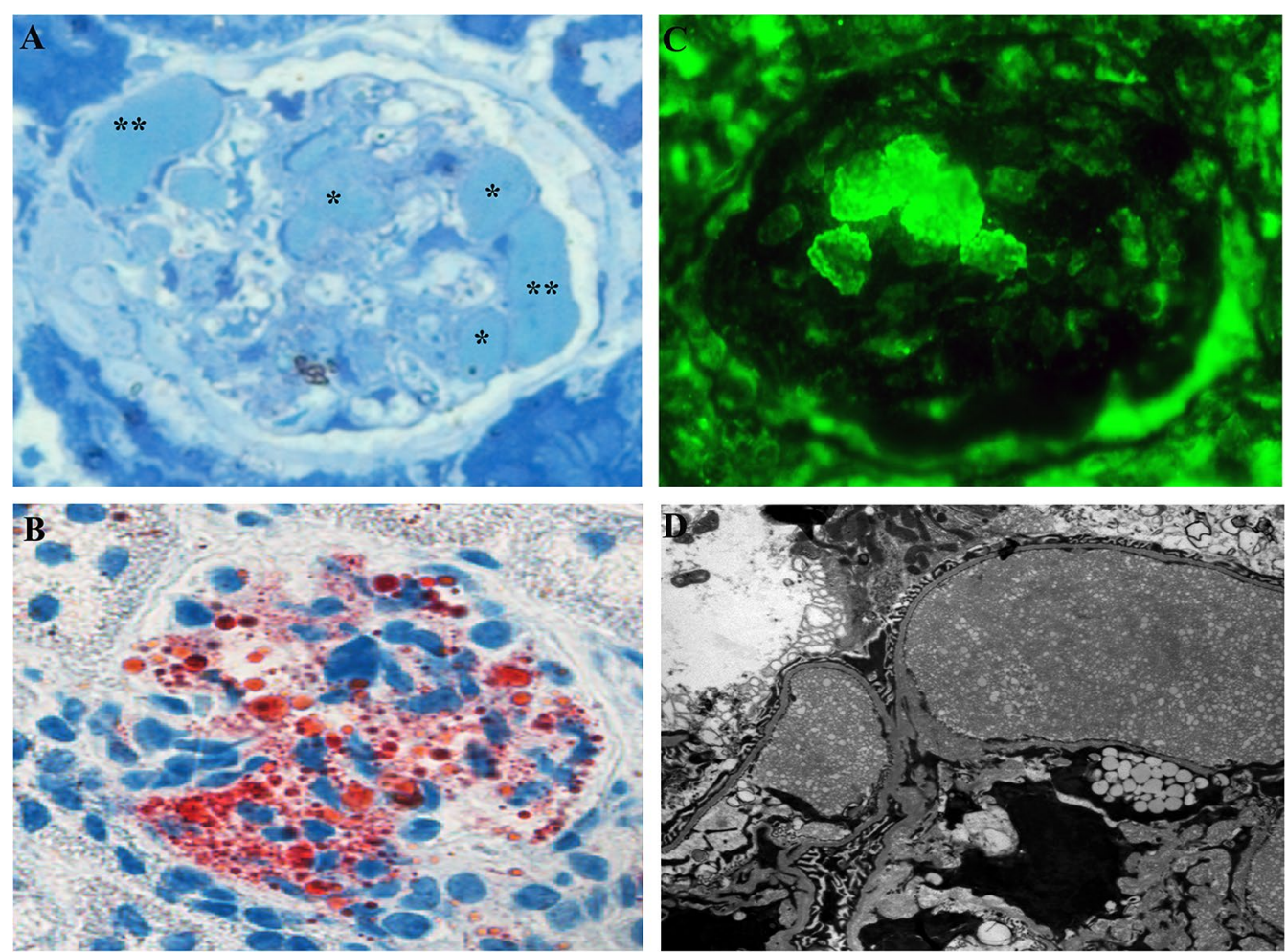

Fig. 4 Typical renal pathologic lesions of lipoprotein glomerulopathy in the murine model. Panel a shows a glomerulus with accumulated lipoprotein thrombi in the dilated lumina of the glomerular capillaries (Toluidine blue staining, $\times 400$ ), ${ }^{*}$ show the lipoprotein thrombi accumulated area. Panel $\mathbf{b}$ shows ApoE primarily in the capillary lumina on immunofluorescence staining (frozen section, $\times 400$ ). Panel c shows an obvious Oil-red O-positive area in the glomerulus ( $\times 400)$. Panel $\mathbf{d}$ shows numerous lipid granules deposited in the dilated capillary lumina of the glomeruli under electron microscope $(\times 6000)$

our study, the lipoprotein thrombi seemed milder in the ApoE Sendai mice than in the ApoE Kyoto mice. Larger samples of LPG model and detailed clinicopathologic analysis of LPG are required to clarify this issue.

LPG is characterized by elevated serum ApoE and hypertriglyceridemia. In the study by Yamamoto, the expression of ApoE Sendai resulted in insufficient normalization of hypercholesterolemia in ApoE-deficient mice and induced high plasma levels of triglyceride [10]. The mechanism of plasma triglyceride induction remains unknown, but it was suggested to be related with the hepatic uptake of plasma cholesterol by the LDL receptor [10]. However, in our study, there was no pulse elevation of plasma triglyceride after virus injection, whereas serum ApoE increased accompanying with decreased total cholesterol concentration. Two weeks after injection, serum ApoE declined along with elevated cholesterol. In contrast to the LPG murine model that was established by Yamamoto et al. our model used younger experiment animal, lower adenovirus injection dosage, and longer observation period. Although we are uncertain if these conditions caused the differences, our work suggested that pulse elevation of plasma triglyceride may not be required for the development of an LPG murine model. In fact, patients with LPG and mild hyperlipidemia have been reported clinically $[5,15]$.

Although the ApoE Kyoto-induced murine LPG model has been founded, the rate of the affected glomeruli was fairly low. Many factors may have contributed to this result. First, adenovirus-mediated expression of ApoE variants can only last for 7-10 days and can immediately decrease thereafter; this was not consistent with the sustained expression of ApoE mutants in human LPG. Second, the function of macrophages in this model seemed intact. Ito et al. [16] showed that impairment of macrophage function resulting from $\mathrm{FcR} \gamma$ deficiency would facilitate the onset of LPG in the presence of the same ApoE abnormalities. Kanamaru et al. [17] presented that LPG-like lesions could be observed in FcR $\gamma$-deficient mice. Usually, in human LPG, foam-like macrophages in the glomeruli are rare. In this study, foam-like macrophages were observed and implied the intact function 

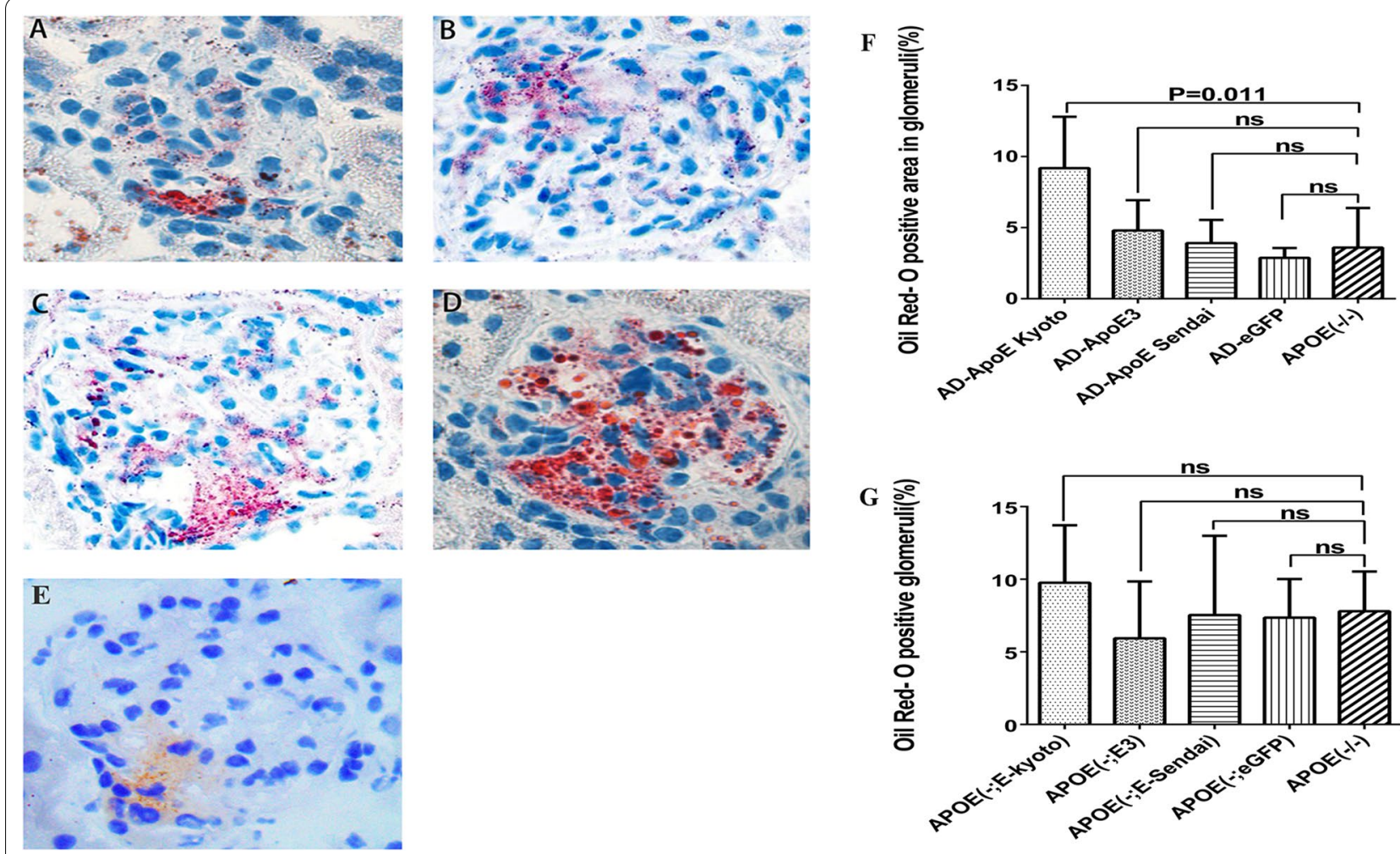

Fig. 5 Oil-red O-positive staining and area analysis of the glomeruli in the five groups. Oil-red O-positive staining in the glomeruli are shown in (a) ApoE (-/-), b AD-eGFP, c AD-ApoE Sendai, d AD-ApoE Kyoto, and e AD-ApoE3. All the Oil-red O-positive glomeruli of five groups were captured. The Oil-red O-positive area was measured by Image I software. We calculated the positive area ratio of each glomerulus using the formula: positive area/total glomerular area, then averaged the ratio, which was shown in (f). The percentage of positive glomeruli was calculated by the formula: number of the positive glomeruli/number of total glomeruli, which was shown in $(\mathbf{g})$

of macrophages. Third, normal fat chow may contribute to the low penetrance of LPG murine model. Defective function of apoE may hinder LDLR-mediated uptake and raise remnant lipoprotein concentration (of VLDL and chylomicrons), which is very sensitive to the diet. More fat chow may place the stress on the aggregation and thrombosis in the kidney. Moreover, the normal ACR of the ApoE variant groups implied that this murine model reflected an early stage of LPG.

Atherosclerosis has been rarely reported among patients with LPG [18], although dyslipidemia commonly persisted. A majority of animal experiments suggested that human apoE3 could postpone the progression of atherosclerosis by decreasing serum total cholesterol or changing the inflammatory cell composition of the local plaque [19-21]. In this study, we found that the atherosclerotic plaque area was lower in the ApoE mutant groups than in the ApoE-deficient mice, which is consistent with Tavori`s work [19]. The similarity of ApoE between human and mouse is about $71 \%$. For mouse, human ApoE is a "mutated ApoE", which may lose a part of function. However, the residual function of the "mutated ApoE" is capable of improving the lipid profiles and playing the protective role against atherosclerosis. It is noticeable that angina pectoris and myocardial infarction were rarely reported in LPG patients, which supports that hypothesis that the mutated ApoE still maintains anti-atherosclerotic effects although it causes LPG meanwhile based on its aggregation prone characteristics [22].

The limitation of this study is the numbers of animals are small. LPG is an autosomal dominant kidney disease with incomplete penetrance. The LPG murine model also showed incomplete penetrance. This makes statistical analysis difficult. It is especially difficult to compare the two pathogenic variants of apoE. Larger sample size of animal model was needed for further study.

\section{Conclusions}

We validated the pathogenicity of ApoE Kyoto in LPG using an animal study. ApoE Kyoto may induce LPG while protecting against atherosclerosis. Further studies are required to clarify the different phenotypes among the ApoE variants. 

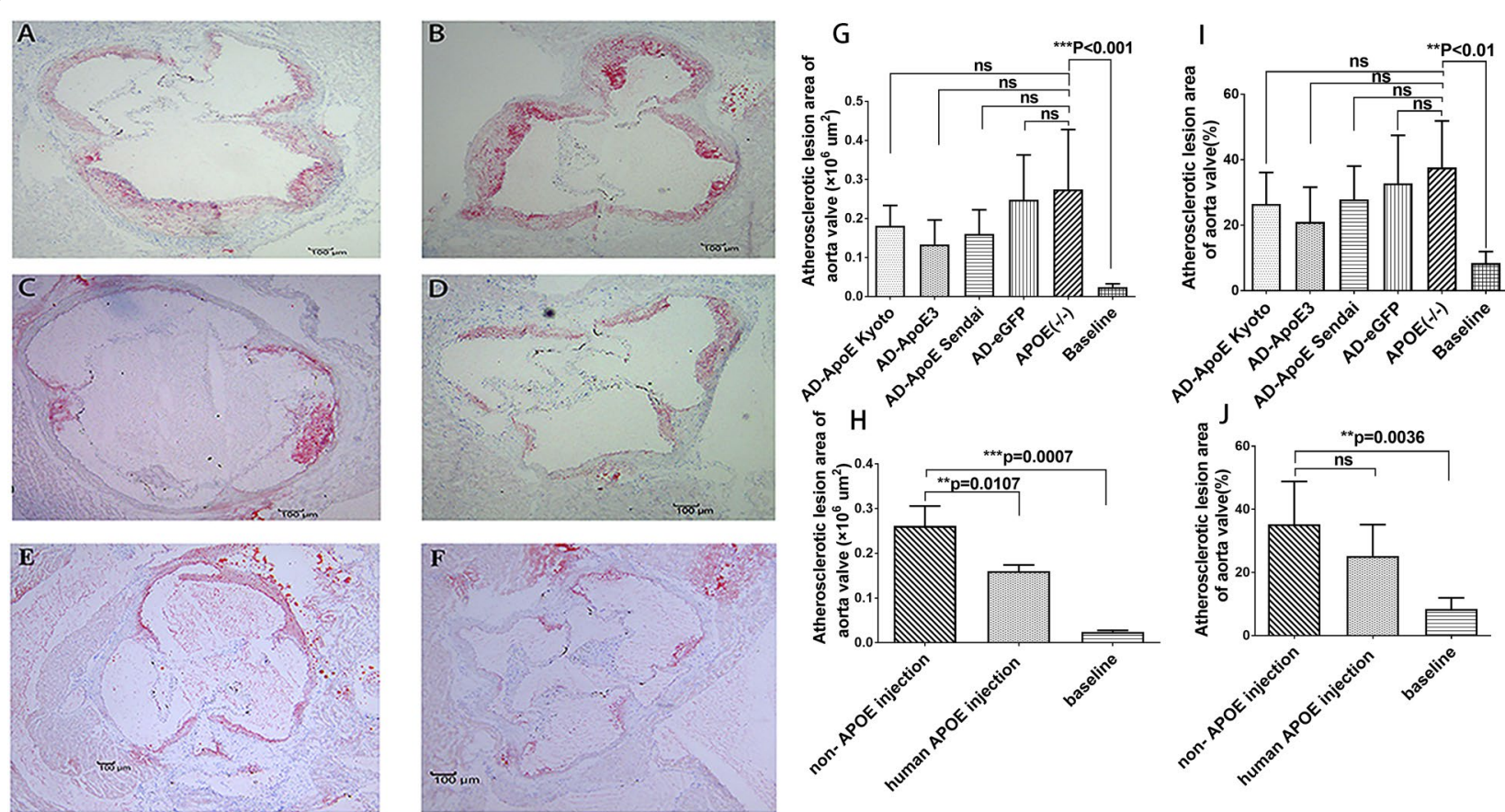

Fig. 6 Oil-red O-positive staining and atherosclerosis plaque area in the aortic valve in the five groups. The atherosclerosis plaque with Oil-red O-positive staining in the aortic valve was observed in 3-month old ApoE (-/-) mice with chow diet and was shown in (f) as baseline group. Three month later, atherosclerosis plaque area of five groups were increased and shown in (a) ApoE (-/-), (b) AD-eGFP, (c) AD-ApoE Sendai, (d) AD-ApoE Kyoto, (e) AD-ApoE3. The atherosclerotic plaque area and its percentage in area difference of aorta adventitia and intima in the aortic valve were quantified by the Motic images Advanced 3.2 software, the ( $\mathbf{g}$ and $\mathbf{h})$ atherosclerosis plaque area and (i and $\mathbf{j})$ percentage of aortic valve level are shown. In Panel ( $\mathbf{h}$ and $\mathbf{j}$ ), the AD-ApoE Sendai, AD-ApoE Kyoto, and AD-ApoE3 groups were pooled as human apoE injection group while the ApoE $(-/-)$ and $A D-e G F P$ groups were pooled as non-APOE injection group

\section{Supplementary Information}

The online version contains supplementary material available at https://doi. org/10.1186/s12967-021-02765-x.

Additional file 1: Table S1. Human ApoE concentration in the cell medium after $48 \mathrm{~h}$ of transfection.

Additional file 2: Figure S1. Oil-red O staining in lung tissue among APOE (-/-) group, APOE(-/Sendai) and APOE(-/Kyoto) groups. Panel $A 、 B 、 C$ showed oil red $O$ staining results in lung from APOE(-/Sendai), $\mathrm{APOE}(-/$ Kyoto) and $\mathrm{APOE}(-/-)$ groups. There are thrombi-like material in pulmonary capillaries. However, there are no differences among them.

\section{Abbreviations}

LPG: Lipoprotein glomerulopathy; ApoE: Apolipoprotein E; LDL: Low-density lipoprotein; LDLR: Low-density lipoprotein receptor; ACR: Albumin-creatinine ratio.

\section{Acknowledgements}

We thank Enago Academy (www.enago.cn) for their help in language editing.

\section{Authors' contributions}

HW completed the project, analyzed data, and wrote the manuscript. JY, ZY and $\mathrm{YL}$ contributed to the animal study. SL and MY contributed to histological preparation. YY participated the design. ZH designed the study, interpreted data and contributed to revising the manuscript. All authors read and approved the final manuscript.

\section{Funding}

This work was supported by grants from the National Natural Science Foundation of China (Grant Number: 81470916). Chengdu Municipal Bureau of Science and Technology (Grant Number: 0040205301D74), and the Sichuan Province Key Technology R\&D Program (Grant Number: 18ZDYF2112).

\section{Availability of data and materials}

The datasets used and/or analyzed during the current study are available from the corresponding author on reasonable request.

\section{Declarations}

\section{Ethics approval and consent to participate}

Our study was approved by the hospital's ethics review board (West China Hospital).

\section{Consent for publication}

Not applicable.

\section{Competing interests}

The authors declare that they have no competing interests.

\section{Author details}

1 Department of Nephrology and National Clinical Research Center for Geriatrics, West China Hospital, Sichuan University, Sichuan, Guoxue Alley, 37\#, Wuhou District, Chengdu 610041, China. ${ }^{2}$ Department of Medical Genetics, West China Hospital, Sichuan University, Chengdu, China. ${ }^{3}$ Department of Pathology, West China Hospital, Sichuan University, Chengdu, China. 
Received: 1 October 2020 Accepted: 22 February 2021

Published online: 04 March 2021

\section{References}

1. Saito T, Matsunaga A, Ito K, Nakashima H. Topics in lipoprotein glomerulopathy: an overview. Clin Exp Nephrol. 2014;18(2):214-7.

2. Hu Z, Huang S, Wu Y, Liu Y, Liu X, Su D, et al. Hereditary features, treatment, and prognosis of the lipoprotein glomerulopathy in patients with the APOE Kyoto mutation. Kidney Int. 2014;85(2):416-24.

3. Saito T, Matsunaga A, Fukunaga M, Nagahama K, Hara S, Muso E. Apolipoprotein E-related glomerular disorders. Kidney Int. 2020;97(2):279-88.

4. Wu H, Yang Y, Hu Z. The novel apolipoprotein E mutation apoE Chengdu (c.518TC, p.L173P) in a Chinese patient with lipoprotein glomerulopathy. J Atheroscler Thromb. 2018;25(8):733-40.

5. Tokura T, Itano S, Kobayashi S, Kuwabara A, Fujimoto S, Horike H, et al. A novel mutation ApoE2 Kurashiki (R158P) in a patient with lipoprotein glomerulopathy. J Atheroscler Thromb. 2011;18(6):536-41.

6. Saito T, Matsunaga A. Significance of a novel apolipoprotein E variant, ApoE Osaka/Kurashiki, in lipoprotein glomerulopathy. J Atheroscler Thromb. 2011:18(6):542-3.

7. Rall SC Jr, Weisgraber KH, Mahley RW. Human apolipoprotein E. The complete amino acid sequence. J Biol Chem. 1982:257(8):4171-8.

8. Wu H, Yang Y, Hu Z. The novel apolipoprotein E mutation ApoE Chengdu (c.518TC, p.L173P) in a Chinese patient with lipoprotein glomerulopathy. J Atheroscler Thromb. 2018:25:733-40.

9. Oikawa S, Matsunaga A, Saito T, Sato H, Seki T, Hoshi K, et al. Apolipoprotein E Sendai (arginine 145->proline): a new variant associated with lipoprotein glomerulopathy. J Am Soc Nephrol. 1997;8(5):820-3.

10. Ishigaki Y, Oikawa S, Suzuki T, Usui S, Magoori K, Kim DH, et al. Virusmediated transduction of apolipoprotein $\mathrm{E}$ (ApoE)-sendai develops lipoprotein glomerulopathy in ApoE-deficient mice. J Biol Chem. 2000;275(40):31269-73.

11. Matsunaga A, Sasaki J, Komatsu T, Kanatsu K, Tsuji E, Moriyama K, et al. A novel apolipoprotein E mutation, E2 (Arg25Cys), in lipoprotein glomerulopathy. Kidney Int. 1999;56(2):421-7.

12. Yang $M$, Weng $Q$, Pan $X$, Hussain HMJ, Yu S, Xu J, et al. Clinical and genetic analysis of lipoprotein glomerulopathy patients caused by APOE mutations. Mol Genet Genomic Med. 2020; e1281.

13. Katsarou M, Stratikos E, Chroni A. Thermodynamic destabilization and aggregation propensity as the mechanism behind the association of apoE3 mutants and lipoprotein glomerulopathy. J Lipid Res. 2018;59(12):2339-48

14. Murano T, Matsumura R, Misawa Y, Ozaki H, Miyashita Y, Yoshida S, et al. Interaction of endothelial cells and triglyceride-rich lipoproteins with apolipoprotein E (Arg25[rarr ]Cys) from a patient with lipoprotein glomerulopathy. Metabolism. 2002;51(2):201-5.

15. Mitani A, Ishigami M, Watase $K$, Minakata T, Yamamura T. A novel apolipoprotein E mutation, ApoE Osaka (Arg158 Pro), in a dyslipidemic patient with lipoprotein glomerulopathy. J Atheroscler Thromb. 2011;18(6):531-5.

16. Ito $K$, Nakashima H, Watanabe M, Ishimura A, Miyahara $Y, A$ be $Y$, et al. Macrophage impairment produced by Fc receptor gamma deficiency plays a principal role in the development of lipoprotein glomerulopathy in concert with apoE abnormalities. Nephrol Dial Transplant. 2012:27:3899-3907.

17. Kanamaru Y, Nakao A, Shirato I, Okumura K, Ogawa H, Tomino Y, et al. Chronic graft-versus-host autoimmune disease in Fc receptor gamma chain-deficient mice results in lipoprotein glomerulopathy. J Am Soc Nephrol. 2002;13(6):1527-33.

18. Matsunaga A, Saito T. Apolipoprotein E mutations: a comparison between lipoprotein glomerulopathy and type III hyperlipoproteinemia. Clin Exp Nephrol. 2014;18(2):220-4.

19. Tavori H, Fan D, Giunzioni I, Zhu L, Linton MF, Fogo AB, et al. Macrophagederived apoESendai suppresses atherosclerosis while causing lipoprotein glomerulopathy in hyperlipidemic mice. J Lipid Res. 2014;55(10):2073-81.

20. Kitajima K, Marchadier DH, Miller GC, Gao GP, Wilson JM, Rader DJ. Complete prevention of atherosclerosis in apoE-deficient mice by hepatic human apoE gene transfer with adeno-associated virus serotypes 7 and 8. Arterioscler Thromb Vasc Biol. 2006;26(8):1852-7.

21. Osman E, Evans V, Graham IR, Athanasopoulos T, Mclntosh J, Nathwani $A C$, et al. Preliminary evaluation of a self-complementary AAV2/8 vector for hepatic gene transfer of human apoE3 to inhibit atherosclerotic lesion development in apoE-deficient mice. Atherosclerosis. 2009;204(1):121-6.

22. Georgiadou D, Stamatakis K, Efthimiadou EK, Kordas G, Gantz D, Chroni A, et al. Thermodynamic and structural destabilization of apoE3 by hereditary mutations associated with the development of lipoprotein glomerulopathy. J Lipid Res. 2013;54(1):164-76.

\section{Publisher's Note}

Springer Nature remains neutral with regard to jurisdictional claims in published maps and institutional affiliations.
Ready to submit your research? Choose BMC and benefit from:

- fast, convenient online submission

- thorough peer review by experienced researchers in your field

- rapid publication on acceptance

- support for research data, including large and complex data types

- gold Open Access which fosters wider collaboration and increased citations

- maximum visibility for your research: over $100 \mathrm{M}$ website views per year

At $\mathrm{BMC}$, research is always in progress.

Learn more biomedcentral.com/submissions 